\title{
ISOMETRIC IMMERSIONS INTO LORENTZIAN PRODUCTS
}

\author{
JULIEN ROTH
}

\begin{abstract}
We give a necessary and sufficient condition for an $n$-dimensional Riemannian manifold to be isometrically immersed into one of the Lorentzian products $\mathbb{S}^{n} \times \mathbb{R}_{1}$ or $\mathbb{H}^{n} \times \mathbb{R}_{1}$. This condition is expressed in terms of its first and second fundamental forms, the tangent and normal projections of the vectical vector field. As applications, we give an equivalent condition in a spinorial way and we deduce the existence of a one-parameter family of isometric maximal deformation of a given maximal surface obtained by rotating the shape operator.
\end{abstract}

\section{INTRODUCTION}

A fundamental question in the theory of submanifolds is to know when a (pseudo)-Riemannian manifold can be isometrically immersed in a given ambient space. In the case where the ambient space is a Riemannian space form, it is a well-known fact that the Gauss, Codazzi and Ricci equations are necessary and sufficient (see [4, 15]). In particular for immersions of codimension 1, the Ricci equation is trivial and the Gauss and Codazzi equations are equivalent to the existence of a local isometric immersion into the desired space form. This fact is also true for psuedoRiemannian manifolds (see [12]). Namely, let $\left(\bar{M}^{n+1}, \bar{g}\right)$ be an orientable psuedo-Riemannian manifold and $(M, g)$ a hypersurface of $\bar{M}$. We denote by $\bar{\nabla}$ and $\nabla$ the Levi-Civita connections of $\left(\bar{M}^{n+1}, \bar{g}\right)$ and $(M, g)$ respectively and by $\bar{R}$ and $R$ the associated curvature tensors. The shape operator of $M$ associated with the normal unit vector field $\nu$ is the symmetric $(1,1)$-tensor $S$ defined by $S X=-\bar{\nabla} \nu$. Then, it is well-known that the following equations holds for any vector fields $X, Y, Z$ tangent to $M$ :

$$
\begin{gathered}
R(X, Y) Z=\bar{R}(X, Y) Z+\delta(g(S X, Z) S Y-g(S Y, Z) S X), \\
\nabla_{X} S Y-\nabla_{Y} S X-S[X, Y]=\bar{R}(X, Y) \nu,
\end{gathered}
$$

where $\delta=1$ is $\nu$ is space-like and $\delta=-1$ if $\nu$ is time-like. These two equations are respectively the Gauss and Codazzi equations. In the case where the ambient manifold is a space form of curvature $\kappa$, they become

$$
\begin{gathered}
R(X, Y) Z=\kappa(g(X, Z) Y-g(Y, Z) X)+\delta(g(S X, Z) S Y-g(S Y, Z) S X), \\
\nabla_{X} S Y-\nabla_{Y} S X-S[X, Y]=0 .
\end{gathered}
$$

Thus, the Gauss and Codazzi equations are defined intrinsically on $\mathrm{M}$ since they involve only the first and second fundamental forms. Moreover, it is well-known that these two equations are also sufficient conditions for $(M, g)$ to be immersed locally and isometrically into the space form of curvature $\kappa$ and compatible signature with given fundamental forms $g$ and $S$.

In the general case, the Gauss and Codazzi equations are not intrinsic because of the curvature tensor of the ambient manifold. Nevertheless, in some special cases, for instance when the ambient space is a Riemannian product of a space form with a real line $\mathbb{M}^{n} \times \mathbb{R}$ or a 3 -dimensional homogenous space, the Gauss and Codazzi equations can be expressed in an extrinsic way. Precisely, for a hypersurface of $\mathbb{M}^{n} \times \mathbb{R}$, the Gauss and Codazzi equations depend only on the first and second fundamental form, the projection $T$ of the vertical vector $\partial_{t}$ onto $T M$ and its normal component $f=\left\langle\partial_{t}, \nu\right\rangle$. In [5], Daniel proved that these two equations together with two more equations give

Date: October 22, 2010.

2000 Mathematics Subject Classification. 53C27, 53C40, 53C $80,58 \mathrm{C} 40$.

Key words and phrases. Isometric Immersions, Gauss and Codazzi Equations, Maximal surfaces, Spinors. 
a necessary and sufficient condition for a manifold $M$ to be immersed locally and isometrically into $\mathbb{M}^{n} \times \mathbb{R}$. Namely, he showed the following

Theorem (Daniel [5]). Let $(M, g)$ be an oriented, simply connected surface and $\nabla$ its Riemannian connection. Let $S$ be field of symmetric endomorphisms $S_{y}: T_{y} M \longrightarrow T_{y} M, T$ a vector field on $M$ and $f$ a smooth function on $M$, such that $\|T\|^{2}+f^{2}=1$. If $(M, g, S, T, f)$ satisfies the Gauss and Codazzi equations for $\mathbb{M}^{n}(\kappa) \times \mathbb{R}$ and fro all $X \in \mathfrak{X}(M)$,

$$
\nabla_{X} T=F S X \quad \text { and } \quad d f(X)=-\langle S X, T\rangle,
$$

then, there exists an isometric immersion

$$
F: M \longrightarrow \mathbb{M}^{2}(\kappa) \times \mathbb{R}
$$

so that the Weingarten operator of the immersion related to the normal $\nu$ is

$$
d F \circ S \circ d F^{-1}
$$

and such that

$$
\partial_{t}=d F(T)+f \nu .
$$

Moreover, this immersion is unique up to a global isometry of $\mathbb{M}^{n}(\kappa) \times \mathbb{R}$ which preserves the orientation of $\mathbb{R}$.

Remark 1. Note that contrary to the case of space forms, the Gauss and Codazzi equations are not sufficient here. Indeed, the compatiblity equations require two additional equtions coming form the fact that $\partial_{t}$ is parallel.

The first aim of this paper is to give such a fundamental theorem of hypersyrfaces for the case of the Lorentzian products $\mathbb{M}^{n}(\kappa) \times \mathbb{R}_{1}$. We prove the following

Theorem 1. Let $(M, g)$ be an oriented, simply connected Riemannian surface and $\nabla$ its Riemannian connection. Let $S$ be field of symmetric endomorphisms $S_{y}: T_{y} M \longrightarrow T_{y} M, T$ a vector field on $M$ and $f$ a smooth function on $M$, such that $\|T\|^{2}-f^{2}=-1$. If $(M, g, S, T, f)$ satisfies the Gauss and Codazzi equations for $\mathbb{M}^{n}(\kappa) \times \mathbb{R}_{1}$ and the following equations

$$
\nabla_{X} T=f S X, \quad d f(X)=\langle S X, T\rangle
$$

then, there exists an isometric immersion

$$
F: M \longrightarrow \mathbb{M}^{n}(\kappa) \times \mathbb{R}_{1}
$$

so that the Weingarten operator of the immersion related to the time-like normal $\nu$ is

$$
d F \circ S \circ d F^{-1}
$$

and such that

$$
\partial_{t}=d F(T)+f \nu .
$$

Moreover, this immersion is unique up to a global isometry of $\mathbb{M}^{n}(\kappa) \times \mathbb{R}_{1}$ which preserves the orientation of $\mathbb{R}$ in the product.

For this, we use the fairly standard method based on differential forms first used by Cartan and after by Tenenblat [15] or Daniel [5] for instance. Then, from Theorem 1, we prove the existence of a one-parameter family of isometric maximal deformations of a given maximal surface into one of the above Lorentzian product.

Finally, we give a spinorial version of this theorem as in the Riemannian case [6, 11, 14] and generalzing the results of [8, 9] to 3-dimensional Lorentzian products.

\section{Preliminaries}

First of all, we fix our conventions for index and summation. Latin letters, $i, j, k$ are integers between 1 and $n$ whereas Greek letters denote integer between 0 and $n+1$. Therefore, for summations, we have $\sum_{\alpha} u_{\alpha}=u_{0}+\cdots u_{n+1}$ and $\sum_{j} u_{j}=u_{1}+\cdots u_{n}$. 
2.1. The compatiblity equations. Let $\left(M^{n}, g\right)$ be an oriented Riemannian hypersurface of $\mathbb{M}^{n}(\kappa) \times \mathbb{R}_{1}$ with normal unit vector field $\nu$. We denote by $\partial_{t}$ the vertical vector of $\mathbb{M}^{n}(\kappa) \times \mathbb{R}_{1}$, that is, the unit vector field giving the orientation of $\mathbb{R}_{1}$ in $\mathbb{M}^{n}(\kappa) \times \mathbb{R}_{1}$. The projection of $\partial_{t}$ on $T M$ is denoted by $T$ and its normal part is $f$. Therefore, we have $\partial_{t}=T+f \nu$, with $f=-\left\langle\partial_{t}, \nu\right\rangle$ since $\langle\nu, \nu\rangle=-1$. We can compute the curvature tensor of $\mathbb{M}^{n}(\kappa) \times \mathbb{R}_{1}$ for vector fields tangent to $M$. We have the following:

Proposition 2.1. For any vector fields $X, Y, Z, W \in \Gamma(T M)$, we have

$$
\begin{aligned}
\langle\bar{R}(X, Y) Z, W\rangle= & \kappa(\langle X, Z\rangle\langle Y, W\rangle-\langle Y, Z\rangle\langle X, W\rangle \\
& +\langle Y, T\rangle\langle W, T\rangle\langle X, Z\rangle-\varepsilon\langle X, T\rangle\langle Z, T\rangle\langle Y, W\rangle \\
& -\langle X, T\rangle\langle W, T\rangle\langle Y, Z\rangle+\varepsilon\langle Y, T\rangle\langle Z, T\rangle\langle X, W\rangle)
\end{aligned}
$$

and

$$
\langle\bar{R}(X, Y) \nu, Z\rangle=-f \kappa(\langle Y, W\rangle\langle X, T\rangle-\langle X, W\rangle\langle Y, T\rangle)
$$

Proof : Let $X, Y, Z, W \in \Gamma(T M)$. We can write these vector fields as follows:

$$
X=\widetilde{X}+x \partial_{t}, Y=\widetilde{Y}+y \partial_{t}, Z=\widetilde{Z}+z \partial_{t}, W=\widetilde{W}+w \partial_{t},
$$

with $\widetilde{X}, \widetilde{Y}, \widetilde{Z}$ and $\widetilde{W}$ tangent to $\mathbb{M}^{n}(\kappa)$ and $x, y, z$ and $w$ some real-valued functions.

Since, $\partial_{t}$ is parallel, we have

$$
\begin{aligned}
& \langle\bar{R}(X, Y) Z, W\rangle=\left\langle\bar{R}_{\mathbb{M}^{n}}(\widetilde{X}, \widetilde{Y}) \widetilde{Z}, \widetilde{W}\right\rangle \\
& =\kappa(\langle\widetilde{X}, \widetilde{Z}\rangle\langle\widetilde{Y}, \widetilde{W}\rangle-\langle\widetilde{Y}, \widetilde{Z}\rangle\langle\widetilde{X}, \widetilde{W}\rangle) \\
& =\kappa\left(\left\langle X-x \partial_{t}, Z-z \partial_{t}\right\rangle\left\langle Y-y \partial_{t}, W-w \partial_{t}\right\rangle\right. \\
& \left.-\left\langle Y-y \partial_{t}, Z-z \partial_{t}\right\rangle\left\langle X-x \partial_{t}, W-w \partial_{t}\right\rangle\right)
\end{aligned}
$$

Moreover, since the vector fields $X, Y, Z$ and $W$ are tangent to $M$ and $\left\langle\partial_{t}, \partial_{t}\right\rangle=-1$, we have $x=-\langle X, T\rangle, y=-\langle Y, T\rangle, z=-\langle Z, T\rangle$ and $w=-\langle W, T\rangle$. So, a straightforward computation yields the first identity.

For the second identity, we denote, $\nu=\widetilde{\nu}+n \partial_{t}$ with $\widetilde{\nu}$ tangent to $\mathbb{M}^{n}(\kappa)$. Clearly, we see that $n=-\left\langle\nu, \partial_{t}\right\rangle=f$. So, we have

$$
\begin{aligned}
\langle\bar{R}(X, Y) \nu, W\rangle= & \left\langle\overline{R_{\mathbb{M}^{n}}}(\widetilde{X}, \widetilde{Y}) \widetilde{\nu}, \widetilde{W}\right\rangle \\
= & \kappa(\langle\widetilde{X}, \widetilde{\nu}\rangle\langle\widetilde{Y}, \widetilde{W}\rangle-\langle\widetilde{Y}, \widetilde{\nu}\rangle\langle\widetilde{X}, \widetilde{W}\rangle) \\
= & \kappa\left(\left\langle X-x \partial_{t}, \nu-f \partial_{t}\right\rangle\left\langle Y-y \partial_{t}, W-w \partial_{t}\right\rangle\right. \\
& \left.-\left\langle Y-y \partial_{t}, \nu-f \partial_{t}\right\rangle\left\langle X-x \partial_{t}, W-w \partial_{t}\right\rangle\right)
\end{aligned}
$$

which gives easily the wanted identity.

Now, using again the fact that the vector field $\partial_{t}$ is parallel, we get the following identities.

Proposition 2.2. For any $X \in \mathfrak{X}(M)$, we have

(1) $\nabla_{X} T=f S X$,

(2) $d f(X)=\langle S X, T\rangle$.

Proof : Since $\partial_{t}=T+f \nu$ is parallel, we have

$$
\begin{aligned}
0 & =\bar{\nabla}_{X} \partial_{t}=\bar{\nabla}_{X} T+d f(X) \nu+f \bar{\nabla}_{X} \nu \\
& =\nabla_{X} T-\left\langle\bar{\nabla}_{X} T, \nu\right\rangle \nu+d f(X) \nu+f \bar{\nabla}_{X} \nu
\end{aligned}
$$

But, by definition, $\bar{\nabla}_{X} \nu=-S X$, so by identification of normal and tangential parts, we have $\nabla_{X} T=f S X$ and $d f(X)=\left\langle\bar{\nabla}_{X} T, \nu\right\rangle=-\left\langle\bar{\nabla}_{X} \nu, T\right\rangle=\langle T, S X\rangle$. This concludes the proof. 
2.2. Moving frames. The main ingeredient to prove Theorem 1 is the technic of moving frames. This tool is fairly standard and one can refer for instance to [16, 15, 13, 5].

Let $\left(M^{n}, g\right)$ be a Riemannian manifold, $\nabla$ its Levi-Civita connection and $R$ its curvature tensor. We consider a field of symmetric operators $S$ on $M$. Let $\left\{e_{1}, \ldots, e_{n}\right\}$ be a local orthonormal frame on $M$ and $\left\{\omega^{1}, \cdots, \omega^{n}\right\}$ its dual basis, $i ., e ., \omega^{i}\left(e_{k}\right)=\delta_{k}^{i}$. We also define $\omega^{n+1}=0$ and the forms $\omega_{j}^{i}, \omega_{j}^{n+1}, \omega_{n+1}^{i}$ and $\omega_{n+1}^{n+1}$ by

$$
\omega_{j}^{i}\left(e_{k}\right)=\left\langle\nabla_{e_{k}} e_{j}, e_{i}\right\rangle, \quad \omega_{j}^{n+1}\left(e_{k}\right)=\left\langle S\left(e_{k}\right), e_{j}\right\rangle, \quad \omega_{n+1}^{j}=-\omega_{j}^{n+1} \text { and } \omega_{n+1}^{n+1}=0 .
$$

Obviously, we have

$$
\nabla_{e_{k}} e_{j}=\sum_{i} \omega_{j}^{i}\left(e_{k}\right) e_{i} \quad \text { and } \quad S e_{k}=\sum_{j} \omega_{j}^{n+1}\left(e_{k}\right) e_{j}
$$

Finally, we denote $R_{k l j}^{i}=\left\langle R\left(e_{k}, e_{l}\right) e_{j}, e_{i}\right\rangle$. Then, we have the following well-known structure forumlas.

Proposition 2.3. We have

$$
\begin{gathered}
d \omega^{i}+\sum_{a} \omega_{a}^{i} \wedge \omega^{a}=0 \\
\sum_{a} \omega_{a}^{n+1} \wedge \omega^{a}=0 \\
d \omega_{j}^{i}+\sum_{a} \omega_{a}^{i} \wedge \omega_{j}^{a}=-\frac{1}{2} \sum_{k} \sum_{l} R_{k l j}^{i} \omega^{k} \wedge \omega^{l} . \\
d \omega_{j}^{n+1}+\sum_{a} \omega_{a}^{n+1} \wedge \omega_{j}^{a}=-\frac{1}{2} \sum_{k} \sum_{l}\left\langle\nabla_{e_{k}} S e_{l}-\nabla_{e_{l}} S e_{k}-S\left[e_{k}, e_{l}\right], e_{j}\right\rangle \omega^{k} \wedge \omega^{l} .
\end{gathered}
$$

These formulas are well-known. One can see [5] for a complete proof of these proposition.

2.3. Other facts about $\mathbb{M}^{n}(\kappa) \times \mathbb{R}_{1}$ and their hypersurfaces. In all this section, we will consider $\left(M^{n}, g\right)$ a hypersurface of $\mathbb{M}^{n}(\kappa) \times \mathbb{R}_{1}$. I We denote by $\mathbb{R}_{1}^{p}$ the $p$-dimensional Lorentz space, that is, $\mathbb{R}^{p}$ endowed with the metric

$$
\left(\mathrm{d} x_{0}\right)^{2}+\cdots+\left(\mathrm{d} x_{p-2}\right)^{2}-\left(\mathrm{d} x^{p-1}\right)^{2} .
$$

and $\mathbb{R}_{2}^{p}$ the $p$-dimensional pseudo-Euclidean space of signature $(p-2,2)$, that is, $\mathbb{R}^{p}$ endowed with the metric

We have the following inclusions

$$
-\left(\mathrm{d} x_{0}\right)^{2}+\left(\mathrm{d} x_{1}\right)^{2}+\cdots+\left(\mathrm{d} x^{p-2}\right)^{2}-\left(d x^{p-1}\right)^{2} .
$$

$$
\mathbb{S}^{n} \times \mathbb{R}_{1} \subset \mathbb{R}^{n+1} \times \mathbb{R}_{1}=\mathbb{R}_{1}^{n+2} \text { and } \mathbb{H}^{n} \times \mathbb{R}_{1} \subset \mathbb{R}_{1}^{n+1} \times \mathbb{R}_{1}=\mathbb{R}_{2}^{n+2},
$$

where $\mathbb{S}^{n}$ and $\mathbb{H}^{n}$ are the respectively the sphere and the hyperbolic space of dimension $n$ defined by

$$
\begin{gathered}
\mathbb{S}^{n}=\left\{\left(x^{0}, \cdots, x^{n}\right) \in \mathbb{R}^{n+1} \mid\left(x^{0}\right)^{2}+\cdots+\left(x^{n}\right)^{2}=1\right\}, \text { and } \\
\mathbb{H}^{n}=\left\{\left(x^{0}, \cdots, x^{n}\right) \in \mathbb{R}_{1}^{n+1} \mid-\left(x^{0}\right)^{2}+\cdots+\left(x^{n}\right)^{2}=-1 ; x^{0}>0\right\} .
\end{gathered}
$$

Thus, we set $\mathbb{E}^{n+2}=\mathbb{R}_{1}^{n+2}$ if $\kappa=1$ and $\mathbb{E}^{n+2}=\mathbb{R}_{2}^{n+2}$ if $\kappa=-1$. With these notation, we have $\mathbb{M}^{n}(\kappa) \times \mathbb{R}_{1} \in \mathbb{E}^{n+2}$.

Now, let $\left(M^{n}, g\right)$ a Riemannian hypersurface of $\mathbb{M}^{n}(\kappa) \times \mathbb{R}_{1}$. We denote respectively by $\nabla, \bar{\nabla}$ and $\overline{\bar{\nabla}}$ the Levi-Civita connections of $M, \mathbb{M}^{n}(\kappa) \times \mathbb{R}_{1}$ and $\mathbb{E}^{n+2}$. We consider $\bar{\nu}$ the unit normal to $\mathbb{M}^{n}(\kappa) \times \mathbb{R}_{1}$ into $\mathbb{E}^{n+2}$, that is,

$$
\bar{\nu}(x)=\left(x^{0}, x^{1}, \cdots, x^{n}, 0\right),
$$

and $\nu$ the unit normal to $M$ into $\mathbb{M}^{n}(\kappa) \times \mathbb{R}_{1}$. We denote by $\bar{S}$ and $S$ the associated shape operators, that is, $\bar{S}$ is defined by

$$
\overline{\bar{\nabla}}_{X} Y=\bar{\nabla}_{X} Y+<\bar{S} X, Y>\bar{\nu}
$$


i.e., $\bar{S} X=-\kappa \overline{\bar{\nabla}}_{X} \bar{\nu}$. Note that the sign $\kappa$ comes for the fact that we have $<\bar{\nu}, \bar{\nu}>=\kappa$.

Let $\left\{e_{1}, \ldots, e_{n}\right\}$ be a local orthonormal frame on $M, e_{n+1}=\nu$ and $e_{0}=\bar{\nu}$. We consider the forms $\omega_{j}^{i}, \omega_{i}^{j}, \omega_{n+1}^{i}$ and $\omega_{n+1}^{n+1}$ as above and in addition, we set

$$
\omega_{\gamma}^{0}\left(e_{k}\right)=<\bar{S} e_{k}, e_{\gamma}>, \quad \text { and } \quad \omega_{0}^{\gamma}=-\kappa \omega_{\gamma}^{0} .
$$

Since $\bar{S}_{X}=-\kappa \overline{\bar{\nabla}}_{X} \bar{\nu}=\kappa\left(-X-\left\langle X, \partial_{t}\right\rangle \partial_{t}\right)$, we have

$$
\omega_{\gamma}^{0}\left(e_{k}\right)=-\kappa\left\langle e_{k}, e_{\gamma}\right\rangle+\kappa \varepsilon\left\langle e_{k}, \partial_{t}\right\rangle\left\langle e_{\gamma}, \partial_{t}\right\rangle .
$$

Hence, by definition, we have $\overline{\bar{\nabla}}_{e_{k}} e_{\beta}=\sum_{\alpha} \omega_{\beta}^{\alpha}\left(e_{k}\right) e_{\alpha}$.

Now, let $\left\{E_{0}, \cdots, E_{n+1}\right\}$ be a frame of $\mathbb{E}^{n+2}$ so that $\left\langle E_{0}, E_{0}\right\rangle=\kappa,\left\langle E_{j}, E_{j}\right\rangle=1$ for $j \in\{1, \cdots, n\}$ and $E_{n+1}=\partial_{t}$ (hence, $\left\langle E_{n+1}, E_{n+1}\right\rangle=-1$ ). We denote by $A \in \mathcal{M}_{n+2}(\mathbb{R})$ the matrix whose columns are the coordinates of the vectors $e_{\beta}$ in the frame $\left\{E_{0}, \cdots, E_{n+1}\right\}$, i.e., $e_{\beta}=\sum_{\alpha} A_{\beta}^{\alpha} E_{\alpha}$. Hence, we have

$$
\overline{\bar{\nabla}}_{e_{k}} e_{\beta}=\sum_{\alpha} \mathrm{d} A_{\beta}^{\alpha} E_{\alpha}
$$

But, on the other hand, we have

$$
\overline{\bar{\nabla}}_{e_{k}} e_{\beta}=\sum_{\alpha, \gamma} \omega_{\beta}^{\gamma}\left(e_{k}\right) A_{\gamma}^{\alpha} E_{\alpha}
$$

which means that $A^{-1} \mathrm{~d} A=\Omega$, with $\Omega=\left(\omega_{\beta}^{\alpha}\right)$.

We set $G=\operatorname{diag}(\kappa, 1, \cdots, 1,-1) \in \mathcal{M}_{n+2}(\mathbb{R})$. We define the two following sets

$$
\mathcal{S}\left(\mathbb{E}^{n+2}\right)=\left\{\left.Z \in \mathcal{M}_{n+2}(\mathbb{R})\right|^{t} Z G Z=G, \operatorname{det} Z=1\right\},
$$

and

$$
\mathfrak{s}\left(\mathbb{E}^{n+2}\right)=\left\{\left.H \in \mathcal{M}_{n+2}(\mathbb{R})\right|^{t} H G+G H=0,\right\} \cong \mathfrak{s o}\left(\mathbb{E}^{n+2}\right) .
$$

Moreover, we set $\mathcal{S}^{+}\left(\mathbb{E}^{n+2}\right)$ the connected component of the identity in $\mathcal{S}\left(\mathbb{E}^{n+2}\right)$. Thus, one can see easily that $A \in \mathcal{S}^{+}\left(\mathbb{E}^{n+2}\right)$ and $\Omega \in \mathfrak{s}\left(\mathbb{E}^{n+2}\right)$.

\section{THE FUNDAMENTAL THEOREM OF HYPERSURFACES}

3.1. The compatibility equations. Let $\left(M^{n}, g\right)$ be a simply connected Riemannian manifold with a field of symmetric operators $S$, a vector field $T$ and a smooth function $f$ on $M$. From the section of preliminaries, we introduce the following definition.

Definition 3.1. We say that $(M, g, S, T, f)$ satisfies the compatibility equation fro $\mathbb{M}^{n}(\kappa) \times \mathbb{R}_{1}$ if $\|T\|^{2}-f^{2}=-1$ and for any $X, Y, Z, W \in \mathfrak{X}(M)$,

$$
\begin{gathered}
R(X, Y) Z,=\langle S Y, Z\rangle X-\langle S X, Z\rangle Y+\kappa(\langle X, Z\rangle Y-\langle Y, Z\rangle X) \\
+\kappa(\langle Y, T\rangle\langle X, Z\rangle T-\langle X, T\rangle\langle Z, T\rangle Y \\
+\langle X, T\rangle\langle Y, Z\rangle T+\langle Y, T\rangle\langle Z, T\rangle X) \\
\nabla_{X} S Y-\nabla_{Y} S X-S[X, Y]=-f \kappa(\langle X, T\rangle Y-\langle Y, T\rangle X) \\
\nabla_{X} T=f S X . \\
d f(X)=\langle S X, T\rangle
\end{gathered}
$$

Remark 2. Note that by differentiating $\|T\|^{2}-f^{2}=-1$, we see that (11) implies (12) at any point where $f$ does not vanish.

Thus, Theorem 1 says that these compatibilty equations are a necessarey and sufficient condition to the existence of a local isometric immersion into $\mathbb{M}^{n}(\kappa) \times \mathbb{R}_{1}$. 
3.2. Proof of Theorem 1. In order to simplify and without lost of generality, we can assume that $\kappa=1$ or $\kappa=-1$. We define $\omega^{i}, \omega^{n+1}, \omega_{j}^{i}, \omega_{j}^{n+1}, \omega_{n+1}^{i}$ and $\omega_{n+1}^{n+1}$ as above and we set

$$
\mathbb{E}^{n+2}= \begin{cases}\mathbb{R}_{1}^{n+2} & \text { if } \kappa>0 \\ \mathbb{R}_{2}^{n+2} & \text { if } \kappa<0\end{cases}
$$

Let $\left\{e_{0}, \cdots, e_{n+1}=\partial_{t}\right\}$ be the canonical frame of $\mathbb{E}$ with $\left|e_{0},\right|^{2}=\kappa,\left|e_{i}\right|^{2}=1$ for $i \in\{1, \cdots, n\}$ and $\left|e_{n+1}\right|^{2}=-1$.

We also consider the following functions $T^{k}=<T, e_{k}>, T^{n+1}=-f$ and $T^{0}=0$, and the following forms: $\omega_{j}^{0}\left(e_{k}\right)=\kappa\left(T^{j} T^{k}+\delta_{j}^{k}\right), \omega_{n+1}^{0}\left(e_{k}\right)=-\kappa f T^{k}, \omega_{0}^{i}=-\kappa \omega_{i}^{0}, \omega_{0}^{n+1}=-\kappa \omega_{n+1}^{0}$ and $\omega_{0}^{0}=0$. Finally, we set $\sigma(X)=<T, X>$, that is, $\sigma=T^{k} \omega^{k}$ and $\Omega=\left(\omega_{\beta}^{\alpha}\right) \in \mathcal{M}_{n+2}(\mathbb{R})$.

Lemma 3.2. We have

$$
\mathrm{d} \sigma=0 .
$$

Proof : Let $X, Y \in \Gamma(T M)$, we have

$$
\begin{aligned}
\mathrm{d} \sigma(X, Y) & =X(\sigma(Y))-Y(\sigma(X))-\sigma([X, Y]) \\
& =<\nabla_{X} T, Y>-<\nabla_{Y} T, X> \\
& =-f<S X, Y>+f<S Y, X> \\
= & 0
\end{aligned}
$$

because $S$ is symmetric.

Lemma 3.3. We have

$$
\mathrm{d} T^{\alpha}=\sum_{\beta} T^{\beta} \omega_{\alpha}^{\beta}
$$

Proof : For $\alpha=0$, we have $T^{0}=0$ and then $\mathrm{d} T^{0}=0$. Moreover, we have

$$
\begin{aligned}
\sum_{\beta} T^{\beta} \omega_{0}^{\beta}\left(e_{k}\right) & =\sum_{j} T^{j} \omega_{0}^{j}\left(e_{k}\right)+T^{n+1} \omega_{0}^{n+1}\left(e_{k}\right) \\
& =\sum_{j} \kappa^{2}\left(T^{j} T^{k}+\delta_{k}^{j}\right) T^{j}+\kappa^{2} f T^{n+1} T^{k} \\
& =\sum_{j}\left(\kappa^{2} T^{j} T^{k} T^{j}\right)+\kappa^{2} T^{k}-\kappa^{2} f^{2} T^{k} \\
& =\kappa^{2} T^{k}\left(\sum_{j}\left(T^{j}\right)^{2}+1-f^{2}\right) \\
& =0 .
\end{aligned}
$$

Indeed, we have, $\sum_{j}\left(T^{j}\right)^{2}-f^{2}=|T|^{2}-f^{2}=-1$.

For $\alpha=n+1$, we have $\mathrm{d} T^{n+1}\left(e_{k}\right)=\mathrm{d} f\left(e_{k}\right)=-<S e_{k}, T>$. On the other hand, we have

$$
\begin{aligned}
\sum_{\beta} T^{\beta} \omega_{n+1}^{\beta}\left(e_{k}\right) & =\sum_{j} T^{j} \omega_{n+1}^{j}\left(e_{k}\right)+T^{\beta} \omega_{n+1}^{n+1}\left(e_{k}\right) \\
& =-\sum_{j} T^{j} \omega_{j}^{n+1}\left(e_{k}\right) \\
& =-<S e_{k}, T> \\
& =\mathrm{d} T^{n+1}\left(e_{k}\right)
\end{aligned}
$$


Finally, for $j \in\{1, \cdots, n\}$,

$$
\begin{aligned}
\mathrm{d} T^{j}\left(e_{k}\right) & =e_{k}<T, e_{j}> \\
& =<\nabla_{e_{k}} T, e_{j}>+<T, \nabla_{e_{k}} e_{j}> \\
& =-f<S e_{k}, e_{j}>+\sum_{i}<T^{i} e_{i}, \nabla_{e_{k}} e_{j}> \\
& =-f \omega_{j}^{n+1}\left(e_{k}\right)+\sum_{i} T^{i} \omega_{j}^{i}\left(e_{k}\right) \\
& =T^{n+1} \omega_{j}^{n+1}\left(e_{k}\right)+\sum_{i} T^{i} \omega_{j}^{i}\left(e_{k}\right) .
\end{aligned}
$$

This achieves the proof.

Lemma 3.4. We have

$$
\mathrm{d} \Omega+\Omega \wedge \Omega=0
$$

Proof : We set $\Phi=d \Omega+\Omega \wedge \Omega$. We recall that $\Omega=\left(\omega_{\beta}^{\alpha}\right)$. Then, from (7), we deduce immediately

$$
\Phi_{i}^{j}=\omega_{n+1}^{i} \wedge \omega_{j}^{n+1}+\omega_{0}^{i} \wedge \omega_{j}^{0}-\frac{1}{2} \sum_{k, l} R_{k l j}^{i} \omega^{k} \wedge \omega^{l} .
$$

But, the Gauss equation (9) is satisfied, that is,

$$
R_{k l j}^{i}=\bar{R}_{k l j}^{i}+\omega_{j}^{n+1}+\wedge \omega_{i}^{n+1}\left(e_{k}, e_{l}\right),
$$

with

$$
\bar{R}_{k l j}^{i}=\kappa\left(\delta_{j}^{k} \delta_{i}^{l}-\delta_{j}^{l} \delta_{i}^{k}+T^{l} T^{i} \delta_{j}^{k}+T^{k} T^{j} \delta_{i}^{l}-T^{k} T^{i} \delta_{j}^{l}-T^{l} T^{j} \delta_{i}^{k}\right) .
$$

Moreover, a straightforward computation gives $\bar{R}_{k l j}^{i}=\omega_{0}^{i} \wedge \omega_{j}^{0}\left(e_{k}, e_{l}\right)$. Thus, from these last four relations, we deduce that $\Phi_{j}^{i}=0$ for any $i, j \in\{1, \cdots, n\}$.

Now, from (8), we have

$$
\Phi_{j}^{n+1}=\frac{1}{2} \sum_{k, l}\left\langle\nabla_{e_{k}} S e_{l}-\nabla_{e_{l}} S e_{k}-S\left[e_{k}, e_{l}\right], e_{j}\right\rangle \omega^{k} \wedge \omega^{l}-\omega_{0}^{n+1} \wedge \omega_{n+1}^{0} .
$$

Since the Coddazzi equation is satisfied, we have

$$
\begin{aligned}
<\nabla_{e_{k}} S e_{l}-\nabla_{e_{l}} S e_{k}-S\left[e_{k}, e_{l}\right], e_{j}> & =-f \kappa\left(T^{k} \delta_{l}^{j}-T^{l} \delta_{k}^{j}\right) \\
& =-\kappa\left(T^{l} T^{n+1} \delta_{k}^{j}-T^{k} T^{n+1} \delta_{l}^{j}\right)
\end{aligned}
$$

Moreover, a simple computation yields $\omega_{0}^{n+1} \wedge \omega_{n+1}^{0}\left(e_{k}, e_{l}\right)=\kappa\left(T^{k} T^{n+1} \delta_{j}^{l}-T^{l} T^{n+1} \delta_{j}^{k}\right)$. Thus, we deduce $\Phi_{j}^{n+1}=0$.

Now, we will show that $\Phi_{n+1}^{0}=0$. First, we have by definition $\omega_{j}^{0}=\kappa\left(T^{j} \sigma+\omega^{j}\right)$. By Lemma 3.2 . we know that $\mathrm{d} \sigma=0$, so we deduce

$$
\mathrm{d} \omega_{j}^{0}=\kappa\left(\mathrm{d} T^{j} \wedge \sigma+\mathrm{d} \omega^{j}\right)=\kappa \mathrm{d} T^{j} \wedge \sigma-1 \kappa \sum_{k} \omega_{k}^{j} \wedge \omega^{k},
$$

where we have used (5). Thus, we get

$$
\begin{aligned}
\Phi_{j}^{0}\left(e_{p}, e_{q}\right)= & \mathrm{d} \omega_{j}^{0}\left(e_{p}, e_{q}\right)+\sum_{k} \omega_{k}^{0} \wedge \omega_{j}^{k}\left(e_{p}, e_{q}\right)+\omega_{n+1}^{0} \wedge \omega_{j}^{n+1}\left(e_{p}, e_{q}\right) \\
= & \kappa\left(\mathrm{d} T^{j}\left(e_{p}\right) \sigma\left(e_{q}\right)-\mathrm{d} T^{j}\left(e_{q}\right) \sigma\left(e_{p}\right)-\omega_{q}^{j}\left(e_{p}\right)+\omega_{p}^{j}\left(e_{q}\right)\right) \\
& +\kappa\left(T^{p} \sum_{k} T^{k} \omega_{j}^{k}\left(e_{q}\right)-T^{q} \sum_{k} T^{k} \omega_{j}^{k}\left(e_{p}\right)+\omega_{j}^{p}\left(e_{q}\right)-\omega_{j}^{q}\left(e_{p}\right)\right) \\
& +\kappa\left(T^{p} T^{n+1} \omega_{j}^{n+1}\left(e_{q}\right)-T^{q} T^{n+1} \omega_{j}^{n+1}\left(e_{p}\right)\right)
\end{aligned}
$$


Since $\sigma\left(e_{p}\right)=T^{p}$ and $\sigma\left(e_{q}\right)=T^{q}$, we conclude by Lemma 3.3 with $\alpha=j$ that $\Phi_{j}^{0}=0$.

Finally, we will prove that $\Phi_{n+1}^{0}=0$. For this, we recall that $\omega_{n+1}^{0}=\kappa T^{n+1} \sigma$, so that, $\mathrm{d} \omega_{n+1}^{0}=$ $\kappa \mathrm{d} T^{n+1} \sigma$. Therefore, we get

$$
\begin{aligned}
\Phi_{n+1}^{0}\left(e_{p}, e_{q}\right)= & \mathrm{d} \omega_{n+1}^{0}\left(e_{p}, e_{q}\right)+\sum_{k} \omega_{k}^{0} \wedge_{n+1}^{k}\left(e_{p}, e_{q}\right) \\
= & \kappa\left(T^{q} \mathrm{~d} T^{n+1}\left(e_{p}\right)-T^{p} \mathrm{~d} T^{n+1}\left(e_{q}\right)\right. \\
& +\kappa\left(T^{p} \sum_{k} T^{k} \omega_{n+1}^{k}\left(e_{q}\right)-T^{q} \sum_{k} T^{k} \omega_{n+1}^{k}\left(e_{p}\right)\right) \\
& +\kappa\left(-\omega_{n+1}^{p}\left(e_{q}\right)+\omega_{n+1}^{q}\left(e_{p}\right)\right) .
\end{aligned}
$$

Using the fact that $S$ is symmetric and Lemma 3.3 with $\alpha=n+1$, we conclude that $\Phi_{n+1}^{0}=0$. To finish, it is obvious from the definition that $\Phi_{0}^{0}=\Phi_{n+1}^{n+1}=0$, and we achieve the proof by noting that $\Phi_{n+1}^{i}=-\Phi_{i}^{n+1}$.

Let $x \in M$, we define $\mathcal{Z}(x)$ as the set of matrices $Z \in \mathcal{S}^{+}\left(\mathbb{E}^{n+2}\right)$ such that the coefficients of the last line of $Z$ are $Z_{\beta}^{n+1}=T^{\beta}(x)$. Then, $\mathcal{Z}(x)$ is a manifold of dimension $\frac{n(n+1)}{2}$. Indeed, this comes from the fact that the map

$$
\begin{array}{ccc}
\widetilde{F}: \mathcal{S}^{+}\left(\mathbb{E}^{n+2}\right) & \longrightarrow & \mathbb{S}\left(\mathbb{E}^{n+2}\right) \\
Z & \longmapsto & \left(Z_{\beta}^{n+1}\right)_{\beta}
\end{array}
$$

with $\mathbb{S}\left(\mathbb{E}^{n+2}\right)=\left\{X \in \mathbb{E}^{n+2} \mid<X, X>=-1\right\}$ is a submersion. Now, we prove the following

Proposition 3.5. Assume that the compatibility equations for $\mathbb{M}^{n}(\kappa) \times \mathbb{R}_{1}$ are fulfiled. Let $x_{0} \in M$ and $A_{0} \in \mathcal{Z}\left(x_{0}\right)$. Then, there exists a neighbourhood $U_{0}$ of $x_{0} \in M$ and a unique map $A: U_{0} \longrightarrow$ $\mathcal{S}^{+}\left(\mathbb{E}^{n+2}\right)$ so that

$$
A^{-1} \mathrm{~d} A=\Omega, \quad A\left(x_{0}\right)=A_{0} \quad \text { and } \quad \forall x \in U_{0}, A(x) \in \mathcal{Z}(x) .
$$

Proof : Let $U$ a neighbourhood of $x_{0}$ in $M$. We set

$$
\mathcal{F}=\left\{(x, Z) \in U \times \mathcal{S}^{+}\left(\mathbb{E}^{n+2}\right) \mid Z \in \mathcal{Z}(x)\right\} .
$$

Obviously, $\mathcal{F}$ is a manifold of dimension $n+\frac{n(n+1)}{2}$ and the tangent space of $\mathcal{F}$ is given by

$$
\mathrm{T}_{(x, Z)} \mathcal{F}=\left\{(u, \xi) \in \mathrm{T}_{x} U \oplus \mathrm{T}_{Z} \mathcal{S}^{+}\left(\mathbb{E}^{n+2}\right) \mid \xi_{\beta}^{n+1}=\left(\mathrm{d} T^{\beta}\right)_{x}(u)\right\} .
$$

Without ambiguity, we denote by $Z$ the projection $U \times \mathcal{S}^{+}\left(\mathbb{E}^{n+2}\right) \longrightarrow \mathcal{S}^{+}\left(\mathbb{E}^{n+2}\right)$, and we consider on $\mathcal{F}$ the matrix of 1 -forms $\Theta=Z^{-1} \mathrm{~d} Z-\Omega$. Thus, for $(x, Z) \in \mathcal{F}$ and $(u, \xi) \in \mathrm{T}_{(x, Z)} \mathcal{F}$, we have $\Theta_{(x, Z)}(u, \xi)=Z^{-1} \xi-\Omega_{x}(u)$. Finally, we set $\mathcal{D}(x, Z)=\operatorname{ker} \Theta_{(x, Z)}$.

Claim: For any $(x, Z) \in \mathcal{F}, \mathcal{D}(x, Z)$ has dimension $n$.

Indeed, first, we note that $\Theta$ belongs to $\mathfrak{s}\left(\mathbb{E}^{n+2}\right)$ as $\Omega$ and $Z^{-1} \mathrm{~d} Z$. In addition, we have by Lemma 3.3

$$
(Z \Theta)_{\beta}^{n+1}=\mathrm{d} Z_{\beta}^{n+1}-\sum_{\gamma} Z_{\gamma}^{n+1} \omega_{\beta}^{\gamma}=\mathrm{d} T^{\beta}-\sum_{\gamma} Z_{\gamma}^{n+1} \omega_{\beta}^{\gamma}=0 .
$$

Hence, $\Theta_{(x, Z)}$ has values in the space $\mathcal{H}=\left\{H \in \mathfrak{s}\left(\mathbb{E}^{n+2}\right) \mid(Z H)_{\beta}^{n+1}=0\right\}$. But, $\mathcal{H}$ is of dimension $\frac{n(n+1)}{2}$ since the map $F$ defined above is a submersion and $H \in \mathcal{H}$ if and only if $Z H \in \operatorname{ker}(\mathrm{d} F)_{Z}$. Moreover, $\{(0, Z H) \mid H \in \mathcal{H}\}$ lies into $\mathrm{T}_{(x, Z)} \mathcal{F}$ and the restriction of $\Theta_{(x, Z)}$ to this subspace is just $(0, Z H) \longmapsto H$. Hence, the map $\Theta_{(x, Z)}$ is onto $\mathcal{H}$, therefore, its rank is $\frac{n(n+1)}{2}$ and so the dimension of its kernel is $n$, which proves the claim. 
Claim: The distribution $\mathcal{D}$ is integrable.

By Lemma 3.4 we have

$$
\begin{aligned}
\mathrm{d} \Theta & =-Z^{-1} \mathrm{~d} Z \wedge Z^{-1} \mathrm{~d} Z-\mathrm{d} \Omega \\
& =-(\Theta+\Omega) \wedge(\Theta+\Omega)-\mathrm{d} \Omega \\
& =-\Theta \wedge \Theta-\Theta \wedge \Omega-\Omega \wedge \Theta .
\end{aligned}
$$

From this, we deduce that if $\xi_{1}, \xi_{2} \in \mathcal{D}$, then $\mathrm{d} \Theta\left(\xi_{1}, \xi_{2}\right)=0$. So, we deduce that

$$
\Theta\left(\left[\xi_{1}, \xi_{2}\right]\right)=\xi_{1} \cdot \Theta\left(\xi_{2}\right)-\xi_{2} \cdot \Theta\left(\xi_{1}\right)-\mathrm{d} \Theta\left(\xi_{1}, \xi_{2}\right)=0 .
$$

So this proves the claim since by Frobenius theorem, $\mathcal{D}$ is integrable.

Now, les $\mathcal{A}$ be the integral manifold through $\left(x_{0}, A_{0}\right)$. If $\xi \in \mathrm{T}_{\left(x_{0}, A_{0}\right)} \mathcal{S}^{+}\left(\mathbb{E}^{n+2}\right)$ is such that $(0, \xi) \in \mathcal{D}\left(x_{0}, A_{0}\right)=\mathrm{T}_{A_{0}} \mathcal{A}$, then $A_{0}^{-1}=\Theta_{\left.x_{0}, A_{0}\right)}(0, \xi)=0$. So $\xi=0$ and so

$$
\mathrm{T}_{\left(x_{0}, A_{0}\right)} \mathcal{A} \cap\left(\{0\} \times \mathrm{T}_{A_{0}} \mathcal{S}^{+}\left(\mathbb{E}^{n+2}\right)\right)=\{(0,0)\} .
$$

Therefore, $\mathcal{A}$ is locally the graph of a function $A: U_{0} \longrightarrow \mathcal{S}^{+}\left(\mathbb{E}^{n+2}\right)$, where $U_{0}$ is a neighbourhood of $x_{0}$ in $U$. Moreover, by construction, this map is unique and satisfies the properties of Proposition 3.5 .

Now, we can prove Theorem 1 using this propostion.

Proof of Theorem 1:

Let $x_{0} \in M, A \in \mathcal{Z}\left(x_{0}\right)$ and $t_{0} \in \mathbb{R}$. Let $\left\{e_{1}, \ldots, e_{n}\right\}$ be a local orthonromal frame of $M$ in a neighbourhood of $x_{0}$. By Proposition 3.5, there exists a unique map $A U_{0} \longrightarrow \mathcal{S}^{+}\left(\mathbb{E}^{n+2}\right)$ satisfying

$$
A^{-1} \mathrm{~d} A=\Omega, \quad A\left(x_{0}\right)=A_{0} \quad \text { and } \quad \forall x \in U_{0}, A(x) \in \mathcal{Z}(x) .
$$

For any $x \in U_{0}$, we set $F^{0}(x)=A_{0}^{0}(x), F^{i}(x)=A_{0}^{i}(x)$ and we consider $F^{n+1}$ the unique function so that $\mathrm{d} F^{n+1}=\varepsilon \sigma$ and $F^{n+1}\left(x_{0}\right)=t_{0}$. Note that $F^{n+1}$ exists because $\mathrm{d} \sigma=0$ and $U_{0}$ is simply connected. Hence, this define a map $F: U_{0} \longrightarrow \mathbb{E}^{n+2}$.

Claim: The map $F$ satisfies all the properties of Theorem 1

First, we show that the image of $U_{0}$ lies into $\mathbb{M}^{n}(\kappa) \times \mathbb{R}_{1}$. Indeed, since $A_{0}^{n+1}=T^{0}=0$ and $A \in S O^{+}\left(\mathbb{E}^{n+1}\right)$, we have,

$$
\kappa\left(F^{0}\right)^{2}+\left(F^{1}\right)^{2}+\sum_{i=2}^{n}\left(F^{i}\right)^{2}=\kappa .
$$

Therefore, $\left(F_{0}, \cdots, F_{n}\right)$ lies into $\mathbb{M}^{n}(\kappa)$ and the values of $F$ lie into $\mathbb{M}^{n}(\kappa) \times \mathbb{R}_{1}$.

Now, since $\mathrm{d} A=A \Omega$, we have for $\alpha \in\{0, \cdots, n+1\}$, we have

$$
\begin{aligned}
\mathrm{d} F^{\alpha}\left(e_{k}\right) & =\sum_{j} A_{j}^{\alpha} \omega_{0}^{j}\left(e_{k}\right)+A_{n+1}^{\alpha} \omega_{0}^{n+1}\left(e_{k}\right) \\
& =\sum_{j} A_{j}^{\alpha}\left(-\delta_{j}^{k}-T^{j} T^{k}\right)-A_{n+1}^{\alpha} T^{n+1} T^{k} \\
& =-A_{k}^{\alpha}-T^{k} \sum_{\beta} A_{\beta}^{\alpha} A_{\beta}^{n+1} \\
& =-A_{k}^{\alpha}
\end{aligned}
$$

and

$$
\mathrm{d} F^{n+1}\left(e_{k}\right)=-\sigma\left(e_{k}\right)=-T^{k}=-A_{k}^{n+1} .
$$

Hence, $\mathrm{d} F\left(e_{k}\right)$ is the opposite the $k$-th column of the matrix $A$. So, since $A$ is invertible, $\mathrm{d} F$ has rank $n$. Thus $F$ is an immersion. Moreover, $A \in \mathcal{S}^{+}\left(\mathbb{E}^{n+2}\right)$, then $<\mathrm{d} F\left(e_{p}\right), \mathrm{d} F\left(e_{q}\right)>=\delta_{q}^{p}$ and $F$ is an isometry.

The columns of $A(x)$ give a direct orthornormal frame of $\mathbb{E}^{n+1}$, and columns 1 to $n$ give a direct orthonromal frame of $\mathrm{T}_{F(x)} F(M)$. The column 0 is the projection of $F(x)$ on $\mathbb{M}^{n} \times\{0\}$, i.e., the 
unit normal $\bar{\nu}(F(x))$ to $\mathbb{M}^{n}(\kappa) \times \mathbb{R}_{1}$ at the point $F(x)$. Therefore, the $(n+1)$-th column is the unit normal $\nu(F(x))$ to $F(M) \operatorname{inM}^{n}(\kappa) \times \mathbb{R}_{1}$ at thr point $F(x)$.

Now, we set $X_{j}=\mathrm{d} F\left(e_{j}\right)$ and we have

$$
\begin{aligned}
<\mathrm{d} X_{j}\left(X_{k}\right), \nu> & =\sum_{\alpha} \mathrm{d} A_{j}^{\alpha}\left(e_{k}\right) A_{n+1}^{\alpha} \\
& =\sum_{\alpha, \beta} A_{\beta}^{\alpha} A_{n+1}^{\alpha} \omega_{j}^{\beta}\left(e_{k}\right) \\
& =\omega_{j}^{n+1}\left(e_{k}\right)=<S e_{k}, e_{j}>
\end{aligned}
$$

that is, the shape operator of $F(M)$ in $\mathbb{M}^{n}(\kappa) \times \mathbb{R}_{1}$ is $\mathrm{d} F \circ S \circ \mathrm{d} F^{-1}$. Finally, the last line of $A$ gives the coordinates of the vertical vector $\partial_{t}$ in the orthornormal frame $\left\{\bar{\nu}, X_{1}, \cdots, X_{n}, \nu\right\}$. Hence, since $A(x) \in \mathcal{Z}(x)$ for $x \in U_{1}$, we have

$$
\partial_{t}=\sum_{j} T^{j} X_{j}+T^{n+1} \nu=\mathrm{d} F(T)-f \nu .
$$

Now, we finish the proof by showing that the local immersion $F$ is unique up to a global isometry of $\mathbb{M}^{n}(\kappa) \times \mathbb{R}_{1}$. For this, let $\widetilde{F}: U_{2} \longrightarrow \mathbb{M}^{n}(\kappa) \times \mathbb{R}_{1}$ be another immersion satisfying all the properties of the theorem, with $U_{2}$ a simply connected neighbourhood of $x_{0}$ included in $U_{0}$ and $\left(\widetilde{X}_{\beta}\right)$ the associated frame, that is, $\widetilde{X}_{j}=\widetilde{F}\left(e_{j}\right), \widetilde{X}_{n+1}$ is the unit normal of $\widetilde{F}(M)$ into $\mathbb{M}^{n}(\kappa) \times \mathbb{R}_{1}$ and $\widetilde{X}_{0}$ is the unit normal to $\mathbb{M}^{n}(\kappa) \times \mathbb{R}_{1}$ into $\mathbb{E}^{n+2}$. Let $\widetilde{A}$ be the matrix of the coordinates of the vectors $\widetilde{X}_{\beta}$ in the frame $\left\{E_{\alpha}\right\}$. Obviously, up to a direct isometry of $\mathbb{M}^{n}(\kappa) \times \mathbb{R}_{1}$, we can assume that $F\left(x_{0}\right)=\widetilde{F}\left(x_{0}\right)$ and the frame $\left(X_{\beta}\right)$ and $\left(\widetilde{X}_{\beta}\right)$ coincide at the point $x_{0}$ and hence $A_{0}\left(x_{0}\right)=\widetilde{A}\left(x_{0}\right)$. Note that this isometry fixes $\partial_{t}$ since the $T^{\alpha}$ are the same. Moreover, these two matrices satisfy all the properties of Proposition 3.5, so by uniqueness of the solution in Proposition 3.5, we have $A_{0}(x)=\widetilde{A}(x)$ for all $x \in M$. Hence, by construction of $F$ and $\widetilde{F}$ from $A$ and $\widetilde{A}$, we deduce that $F=\widetilde{F}$ on $U_{2}$.

Now, we will prove that $F$ can be extended in a unique way to $M$. For his, we consider $x_{1} \in M$ and a curve $\Gamma:[0,1] \longrightarrow M$ so that $\Gamma(0)=x_{0}$ and $\Gamma(1)=x_{1}$. Then, for each point $\Gamma(t)$, there exists a neighbourhood of $\Gamma(t)$ such that there exists an isometric immersion of this neighbouhood into $\mathbb{M}^{n}(\kappa) \times \mathbb{R}_{1}$ satisfying the properties of the theorem. From this family of neighbourhood, we can extract a finite subsequence $\left(V_{0}, \cdots, V_{p}\right)$ covering $\Gamma$ with $V_{0}=U_{0}$. Hence, by uniqueness, we can extend $F$ to $V_{p}$ and define $F\left(x_{1}\right)$.We conclude by noting that since $M$ is simply connected, the value of $F\left(x_{1}\right)$ does not depend on the choice of the curve $\Gamma$.

\section{Application to maximal surfaces: the ASsociate family}

We deduce a first application of Theorem 1, namely the existence of a one-parameter family of maximal conformal immersion associated to a given maximal surface. This result is analogous to the result of B. Daniel for minimal surfaces into Riemannian products $\mathbb{M}^{n}(\kappa) \times \mathbb{R}$. First, we give this elementary proposition.

Proposition 4.1. Assume that $\left(M^{2}, g, S, T, f\right)$ satisfies the compatibility equations for $\mathbb{M}^{2}(\kappa) \times \mathbb{R}_{1}$ and that $S$ is trace-free. For $\theta \in \mathbb{R}$, we define

$$
\begin{aligned}
& S_{\theta}=e^{\theta J} S=\cos (\theta) S+\sin (\theta) J S, \\
& S_{\theta}=e^{\theta J} T=\cos (\theta) S+\sin (\theta) J T .
\end{aligned}
$$

Then $S_{\theta}$ is symmetric, trace-free, $\left\|T_{\theta}\right\|^{2}-f^{2}=-1$, and $\left(M^{2}, g, S_{\theta}, T_{\theta}, f\right)$ satisfies the compatibility equations for $\mathbb{M}^{2}(\kappa) \times \mathbb{R}_{1}$.

Remark 3. Note that $S_{\theta}$ and $T_{\theta}$ are obtained from $S$ and $T$ by a rotation of angle $\theta$.

Proof : The fact that $S_{\theta}$ is symmetric is just coming from the definition and a simple computation shows that $S_{\theta}$ is trace-free. Moreover since $\left.<T, J T\right\rangle=0$, we deduce immediately that $\left\|T_{\theta}\right\|=$ $\|T\|$. Since $e^{J} S$ commutes with $\nabla_{X}$, the conditions $\sqrt{11}$ and $\sqrt{12}$ are fulfiled for $S_{\theta}$ and $T_{\theta}$. Now, 
we will prove that the Gauss and Codazzi equations are satified. First, we remark that on surface, the Gauss equation becomes

$$
K=-\operatorname{det}(S)+\kappa\left(-1-\|T\|^{2}\right)=-\operatorname{det}(S)-\kappa f^{2} .
$$

Since $\operatorname{det}\left(e^{\theta J}\right)=1$, we have $\operatorname{det}\left(S_{\theta}\right)=\operatorname{det}(S)$ and then the Gauss equation.

From this, we deduce the existence of the associated familiy.

Theorem 2. Let $\left(M^{2}, g\right)$ be a simply connected Riemannian surface and $F: M \longrightarrow \mathbb{M}^{2}(\kappa) \times \mathbb{R}_{1}$ a conformal maximal immersion with induced normal $\nu$. Let $S$ be the symmetric operator on $M$ induced by the shape operator of $F$. Let $T$ be the vector field on $M$ so that $\mathrm{d} F(T)$ is the projection of $\partial_{t}$ on $\mathrm{T}(F(M))$, and $f=-<\nu, \partial_{t}>$.

Let $x_{0} \in M$. Then, there exists a unique family $\left(F_{\theta}\right)_{\theta \in \mathbb{R}}$ of conformal maximal immersions of $M$ into $\mathbb{M}^{n}(\kappa) \times \mathbb{R}_{1}$ such that

(1) $F_{\theta}\left(x_{0}\right)=F\left(x_{0}\right)$ and $\left(\mathrm{d} F_{\theta}\right)_{x_{0}}=(\mathrm{d} F)_{x_{0}}$,

(2) the metrics induces on $M$ by $F$ and $F_{\theta}$ are the same,

(3) the symmetric operator on $M$ induce by the shape operator of $F_{\theta}$ is $e^{\theta J} S$,

(4) $\partial_{t}=\mathrm{d} F_{\theta}\left(e^{\theta J} T\right)+f \nu_{\theta}$, where $\nu_{\theta}$ is the unit normal to $F_{\theta}$.

Moreover, $F_{0}=F$ and the family $\left(F_{\theta}\right)_{\theta \in \mathbb{R}}$ is continous with respect to $\theta$.

Proof: Let $g$ be the metric on $M$ induced by $F$. Thus, $(M, g, S, T, f)$ satisfies the compatibily equations for $\mathbb{M}^{2}(\kappa) \times \mathbb{R}_{1}$. So, by Proposition 4.1. $\left(M, g, S_{\theta}, T_{\theta}, f\right)$ also satisfy these compatibility equations. Therefore, by Theorem 1, there exists a unique isometric immersion $F_{\theta}$ satisfying the properties of the theorem. By uniqueness, it is clear that $F_{0}=F$. Moreover, From the proof of Theorem 1. $\left(M, g, S_{\theta}, T_{\theta}, f\right)$ defines for any $\theta$ a matrix of 1-forms $\Omega_{\theta}$ and a matrix of functions $A_{\theta}$ with $A_{\theta}^{-1} \mathrm{~d} A_{\theta}=\Omega_{\theta}$. From the definition of $\Omega_{\theta}$, we get the coninuity of $\Omega_{\theta}$ and hence, of $A_{\theta}$. By the construction of $F_{\theta}$ using only $A_{\theta}$, we deduce that $F_{\theta}$ is also continuous.

\section{Spinorial characterization of Surfaces into $\mathbb{M}^{2}(\kappa) \times \mathbb{R}_{1}$}

The second application of the main result is the spinorial characterization of surfaces into $\mathbb{M}^{2}(\kappa) \times \mathbb{R}_{1}$. It is now well-known that a necessary and sufficient condition to get an isometric immersion of a surface into Euclidean 3-space is existence of a special spinor field called restricted Killing spinor field (see [6, 11]). These results are the geometrically invariant version of previous work on the spinorial Weierstrass representation by U. Abresch, D. Sullivan, R. Kusner, N. Schmidt, and many others (see [7]). This representation was expressed by T. Friedrich ([6]) for surfaces in $\mathbb{R}^{3}$ and then extended to other 3 -dimensional Riemannian manifolds ([11, 14]). In a recent work, we have treated this question for surfaces of arbitrary signature in pseudo-Riemannian 3-dimensional space forms [9].

More generally, the restriction $\varphi$ of a parallel spinor field on $\mathbb{R}^{n+1}$ to an oriented Riemannian hypersurface $M^{n}$ is a solution of a restricted Killing equation

$$
\nabla_{X}^{\Sigma M} \varphi=-\frac{1}{2} \gamma^{M}(S(X)) \varphi
$$

where $\gamma^{M}$ and $\nabla^{\Sigma M}$ are respectively the Clifford multiplication and the spin connection on $M^{n}$, and $S$ is the Weingarten tensor of the immersion. Conversely, Friedrich proves in $\underline{6}$ that, in the two dimensional case, if there exists a restricted Killing spinor field satisfying equation (13), where $S$ is an arbitrary field of symmetric endomorphisms of $T M$, then $S$ satisfies the Gauss and Codazzi equations of hypersurface theory and is consequently the Weingarten tensor of a local isometric immersion of $M$ into $\mathbb{R}^{3}$. Moreover, in this case, the solution $\varphi$ of the restricted Killing equation is equivalently a solution of the Dirac equation

$$
D \varphi=H \varphi,
$$

where $|\varphi|$ is constant and $H$ is a real-valued function.

In the case of immersions of Riemannian surfaces into a Lorentzian space two spinor fields are needed, as we will see in Theorem 3 . 
5.1. Basic facts about spin geometry of hypersurfaces. Here, we recall the basics of spin geometry. For more details, one can refer to [10, 2, 1] for instance.

Let $\left(M^{p, q}, g\right), p+q=2$, be an oriented pseudo-Riemannian surface of arbitrary signature isometrically immersed into a three-dimensional pseudo-Riemannian spin manifold $\left(N^{r, s}, \bar{g}\right)$. We introduce the parameter $\delta$ as follows: $\delta=i$ if the immersion is timelike and $\delta=1$ if the immersion is spacelike. Let $\nu$ be a unit vector normal to $M$. The fact that $M$ is oriented implies that $M$ carries a spin structure induced from the spin structure of $N$ and we have the following identification of the spinor bundles and Clifford multiplications:

$$
\left\{\begin{array}{l}
\Sigma N_{\mid M} \equiv \Sigma M . \\
X \cdot \varphi_{\mid M}=(\delta X \bullet \nu \bullet \varphi)_{\mid M},
\end{array}\right.
$$

where $\cdot$ and $\bullet$ are the Clifford multiplications, respectively on $M$ and $N$. Moreover, we have the following well-known spinorial Gauss formula

$$
\bar{\nabla}_{X} \varphi=\nabla_{X} \varphi+\frac{\delta}{2} S X \cdot \varphi
$$

where $S$ is the shape operator of the immersion. Finally, we recall the Ricci identity on $M$

$$
R\left(e_{1}, e_{2}\right) \varphi=\frac{1}{2} \varepsilon_{1} \varepsilon_{2} R_{1221} e_{1} \cdot e_{2} \cdot \varphi,
$$

where $e_{1}, e_{2}$ is a local orthonormal frame of $M$ and $\varepsilon_{j}=g\left(e_{j}, e_{j}\right)$.

The complex volume element on the surface depends on the signature and is defined by

$$
\omega_{p, q}^{\mathbb{C}}=i^{q+1} e_{1} \cdot e_{2}
$$

Obviously $\left(\omega_{p, q}^{\mathbb{C}}\right)^{2}=1$ independently of the signature and the action of $\omega^{\mathbb{C}}$ splits $\Sigma M$ into two eigenspaces $\Sigma^{ \pm} M$ of real dimension 2. Therefore, a spinor field $\varphi$ can be written as $\varphi=\varphi^{+}+\varphi^{-}$ with $\omega^{\mathbb{C}} \cdot \varphi^{ \pm}= \pm \varphi^{ \pm}$. Finally, we denote $\bar{\varphi}=\omega^{\mathbb{C}} \cdot \varphi=\varphi^{+}-\varphi^{-}$.

\subsection{Special spinor fields.}

5.2.1. Special spinor fields on $\mathbb{M}^{2}(\kappa) \times \mathbb{R}_{1}$. First, we construct particular spinor fields on $\mathbb{M}^{2}(\kappa) \times \mathbb{R}_{1}$ by lifting a Killing spinor of $\mathbb{M}^{2}(\kappa)$ to the product $\mathbb{M}^{2}(\kappa) \times \mathbb{R}_{1}$. For this, we will consider some particular and interesting sections, precisely the sections which do not depend on $t$.

We have the spinorial Gauss formula,

$$
\bar{\nabla}_{X} \varphi=\nabla_{X} \varphi+\frac{i}{2} \gamma(S X) \gamma(\nu) \varphi
$$

where $\bar{\nabla}$ is the spinorial connection on $\mathbb{M}^{2}(\kappa) \times \mathbb{R}_{1}$, the spinorial connection on $\mathbb{M}^{2}(\kappa)$ is $\nabla$, the Clifford multiplication on $\mathbb{M}^{2}(\kappa) \times \mathbb{R}_{1}$ is $\gamma$ and $S$ the shape operator of the immersion of $\mathbb{M}^{2}(\kappa)$ into $\mathbb{M}^{2}(\kappa) \times \mathbb{R}_{1}$. Since $\mathbb{M}^{2}(\kappa)$ is totally geodesic in the product $\mathbb{M}^{2}(\kappa) \times \mathbb{R}_{1}$, we get by taking $\varphi_{t}=\varphi_{0}$ a Killing spinor on $\mathbb{M}^{2}(\kappa)$, i.e. $\nabla_{X} \varphi_{0}=\eta \gamma^{\mathbb{M}^{2}}(X) \varphi_{0}$ :

$$
\bar{\nabla}_{X} \varphi=\eta \gamma^{\mathbb{M}^{2}}(X) \varphi=i \eta \gamma(X) \gamma\left(\partial_{t}\right) \varphi
$$

On the other hand, the complexe volume form $\omega_{\mathbb{C}}=-i e_{1} \cdot e_{2} \cdot \partial_{t}$ acts as identity, so we have

$$
\gamma\left(e_{1}\right) \gamma\left(\partial_{t}\right) \varphi=i \gamma\left(e_{2}\right) \varphi, \text { and } \gamma\left(e_{2}\right) \gamma\left(\partial_{t}\right) \varphi=-i \gamma\left(e_{1}\right) \varphi
$$

So we deduce that

$$
\left\{\begin{array}{l}
\bar{\nabla}_{e_{1}} \varphi=i \eta \gamma\left(e_{2}\right) \varphi, \\
\bar{\nabla}_{e_{2}} \varphi=-i \eta \gamma\left(e_{1}\right) \varphi, \\
\bar{\nabla}_{\partial_{t}} \varphi=0 .
\end{array}\right.
$$

These particular spinor fields are the analogue of Killing spinor field for space forms and will be play a important role in the sequel. 
5.2.2. Restriction to a surface. Now, let $(M, g)$ be a surface of $\mathbb{M}^{2}(\kappa) \times \mathbb{R}$, oriented by $\nu$. Since $M$ is oriented, it could be equiped with a spin structure induce from the spin structure of $\mathbb{M}^{2} \times \mathbb{R}$. Moreover, as we saw, we have the following identification between the spinor bundles

$$
\Sigma\left(\mathbb{M}^{2} \times \mathbb{R}\right)_{\mid N} \cong \Sigma M,
$$

and the spinorial Gauss forumla 15 gives the relation between the spinorial connctions of $M$ and $\mathbb{M}^{2} \times \mathbb{R}$. For any $X \in \mathfrak{X}(M)$ and any $\psi \in \Gamma\left(\Sigma\left(\mathbb{M}^{2} \times \mathbb{R}\right)\right)$, we have

$$
\left(\bar{\nabla}_{X} \psi\right)_{\mid M}=\nabla_{X}\left(\psi_{\mid M}\right)+\frac{i}{2} \gamma^{M}(S X) \psi_{\left.\right|_{M}} .
$$

If we use this forumla for the particular spinor field on $\mathbb{M}^{2} \times \mathbb{R}$ given by (17), we get

$$
\nabla_{X} \varphi=\eta \gamma\left(X_{t}\right) \gamma\left(\partial_{t}\right) \varphi-\frac{i}{2} \gamma^{M}(S X) \varphi
$$

where $X_{t}$ is the part of $X$ tangent to $\mathbb{M}^{2}$, that is,

$$
X_{t}=X-\left\langle X, \partial_{t}\right\rangle \partial_{t}=X-\langle X, T\rangle T-f\langle X, T\rangle \nu
$$

So, we deduce that

$$
\begin{aligned}
\nabla_{X} \varphi & =i \eta \gamma(X) \gamma\left(\partial_{t}\right) \varphi-i \eta\left\langle X, \partial_{t}\right\rangle \gamma\left(\partial_{t}\right) \gamma\left(\partial_{t}\right) \varphi-\frac{i}{2} \gamma^{M}(S X) \varphi \\
& =i \eta \gamma(X) \gamma\left(\partial_{t}\right) \varphi+i \eta\left\langle X, \partial_{t}\right\rangle \varphi-\frac{i}{2} \gamma^{M}(S X) \varphi \\
& =i \eta \gamma(X) \gamma(T) \varphi+i \eta f \gamma(X) \gamma(\nu) \varphi+\eta\langle X, T\rangle \varphi-\frac{i}{2} \gamma^{M}(S X) \varphi
\end{aligned}
$$

On the other hand, if we denote by $\omega=e_{1} \cdot e_{2}$ the real volume element on $M$, we have the following well-known relations (see [9])

$$
\left\{\begin{array}{l}
\gamma(X)=-\gamma^{M}(X) \gamma^{N}(\omega) \\
\gamma(\nu)=-i \gamma^{M}(\omega)
\end{array}\right.
$$

By using these two identities, the fact that $\omega^{2}=-1$ and that $\omega$ anti-commuts with vector fields tangent to $M$, we get

$\nabla_{X} \varphi=i \eta \gamma^{M}(X) \gamma^{M}(\omega) \gamma^{M}(T) \gamma^{M}(\omega) \varphi+i \eta\langle X, T\rangle \varphi-\eta f \gamma^{M}(X) \gamma^{M}(\omega) \gamma^{M}(\omega) \varphi-\frac{i}{2} \gamma^{M}(S X) \varphi$

Now, we can rewrite this equation in an intrinsic way as follows

$$
\nabla_{X} \varphi=i \eta X \cdot T \cdot \varphi+\eta f X \cdot \varphi+i \eta\langle X, T\rangle \varphi-\frac{i}{2} S X \cdot \varphi
$$

where "." stands for the Clifford multiplication on $M$.

\subsection{A spinorial version of the fundamental theorem.}

Theorem 3. Let $(M, g)$ be a connected, oriented and simply connectd Riemannian surface. Let $T$ be a vector field, $f$ and $H$ two real functions on $M$ satisfying $f^{2}-\|T\|^{2}=-1$. The following three data are equivalent:

i) There exist two non-trivial spinor fields $\varphi_{1}$ and $\varphi_{2}$, orthogonal and solution of the equation

$$
\nabla_{X} \varphi=-\frac{i}{2} S X \cdot \varphi+i \eta X \cdot T \cdot \varphi+\eta f X \cdot \varphi+i \eta\langle X, T\rangle \varphi
$$

where $S$ satisfies

$$
\nabla_{X} T=f S X, \quad \text { and } \quad d f(X)=\langle S X, T\rangle .
$$

ii) There exists an isometric immersion $F$ from $M$ into $\mathbb{M}^{2}(\kappa) \times \mathbb{R}_{1}$ of mean curvature $H$, such that the shape operator related to the time-like normal $\nu$ is given by

$$
d F \circ S \circ d F^{-1}
$$

and such that

$$
\partial_{t}=d F(T)+f \nu .
$$


5.4. Restricted Killing fields and compatiblity equations. In a first time, we show that the existence of a restricteded Killing spinor implies the Gauss and Codazzi equations. For this, let $(M, g)$ be an oriented surface with a non-trivial spinor field solution of the equation (18). We will see that the integrability conditions for this equation are precisely the Gauss and Codazzi equations.

Proposition 5.1. Let $(M, g)$ be an oriented surface with $\varphi$ solution of (18) and such that the equations (11) and (12) are satisfied. Then, we have

$$
\left(R_{1212}+\operatorname{det}(S)-\kappa f^{2}\right) \omega \cdot \varphi=i\left(d^{\nabla} S\left(e_{1}, e_{2}\right)+\kappa f J(T)\right) \cdot \varphi,
$$

Proof: The proof of this proposition is based on the computation of the spinorial curvature applied to the spinor field $\varphi$. We recall that the spinorial curvature is defined as follows

$$
\mathcal{R}(X, Y) \varphi=\nabla_{X} \nabla_{Y} \varphi-\nabla_{Y} \nabla_{X} \varphi-\nabla_{[X, Y]} \varphi
$$

for $X, Y \in \mathfrak{X}(M)$. Here, we do the computations for $\varphi$. It is clear that the same computation for $\psi$ with only some changes of sign yields the result. Using the expression given by (18), the equations (11) and (12), the fact that $\omega^{2}=-1$ and that $\omega$ anticommuts with the vector fields tangent to $M$, we get

$$
\begin{aligned}
& \nabla_{X} \nabla_{Y} \varphi=-\underbrace{\frac{\eta}{2} f Y \cdot A X \cdot \varphi}_{\alpha_{1}(X, Y)}-\underbrace{\eta^{2} Y \cdot T \cdot X \cdot T \cdot \varphi}_{\alpha_{2}(X, Y)}+\underbrace{\eta^{2} f Y \cdot T \cdot X \cdot \varphi}_{-\alpha_{3}(X, Y)} \\
& +\underbrace{\frac{\eta}{2} Y \cdot T \cdot A X \cdot \varphi}_{-\alpha_{4}(X, Y)}-\underbrace{\eta\langle A X, T\rangle Y \cdot \varphi}_{-\alpha_{5}(X, Y)}+\underbrace{\eta^{2} f Y \cdot X \cdot T \cdot \varphi}_{-\alpha_{6}(X, Y)} \\
& -\underbrace{\eta^{2}\langle X, T\rangle Y \cdot T \cdot \varphi}_{\alpha_{7}(X, Y)}-\underbrace{\eta^{2} f^{2} Y \cdot X \cdot \varphi}_{-\alpha_{8}(X, Y)}+\underbrace{\eta^{2} f\langle X, T\rangle Y \cdot \varphi}_{-\alpha_{9}(X, Y)} \\
& -\underbrace{i \eta f Y \cdot A X \cdot \varphi}_{-\alpha_{10}(X, Y)}+\underbrace{i \eta f\langle Y, A X\rangle \varphi}_{\alpha_{11}(X, Y)}-\underbrace{\eta^{2}\langle Y, T\rangle X \cdot T \cdot \varphi}_{\alpha_{12}(X, Y)} \\
& +\underbrace{\eta^{2} f\langle Y, T\rangle X \cdot \varphi}_{-\alpha_{13}(X, Y)}-\underbrace{\eta^{2}\langle X, T\rangle\langle Y, T\rangle \varphi}_{\alpha_{14}(X, Y)}+\underbrace{\frac{\eta}{2}\langle Y, T\rangle A X \cdot \varphi}_{-\alpha_{15}(X, Y)} \\
& -\underbrace{\frac{i}{2} \nabla_{X}(A Y) \cdot \varphi}_{-\alpha_{16}(X, Y)}+\underbrace{\frac{\eta}{2} A Y \cdot X \cdot T \cdot \varphi}_{-\alpha_{17}(X, Y)}-\underbrace{\frac{\eta}{2} f A Y \cdot X \cdot \varphi}_{-\alpha_{18}(X, Y)} \\
& +\underbrace{\frac{\eta}{2}\langle X, T\rangle A Y \cdot \varphi}_{-\alpha_{19}(X, Y)}-\underbrace{\frac{1}{4} A Y \cdot A X \cdot \varphi}_{-\alpha_{20}(X, Y)}+\underbrace{i \eta \nabla_{X} Y \cdot T \cdot \varphi}_{\alpha_{21}(X, Y)} \\
& -\underbrace{i \eta f \nabla_{X} Y \cdot \varphi}_{\alpha_{22}(X, Y)}+\underbrace{i \eta\left\langle\nabla_{X} Y, T\right\rangle \varphi}_{\alpha_{23}(X, Y)}
\end{aligned}
$$

That is,

$$
\nabla_{X} \nabla_{Y} \varphi=\sum_{i=1}^{23} \alpha_{i}(X, Y)
$$

Obviously, by symmetry, we have

$$
\nabla_{Y} \nabla_{X} \varphi=\sum_{i=1}^{23} \alpha_{i}(Y, X)
$$


On the other hand, we have

$$
\begin{aligned}
\nabla_{[X, Y] \varphi}= & \underbrace{i \eta[X, Y] \cdot T \cdot \varphi}_{\beta_{1}([X, Y])}-\underbrace{i \eta f[X, Y] \cdot \varphi}_{-\beta_{2}([X, Y])} \\
& +\underbrace{i \eta\langle[X, Y], T\rangle \varphi}_{\beta_{3}([X, Y])}-\underbrace{\frac{i}{2} A[X, Y] \cdot \varphi}_{-\beta_{4}([X, Y])} .
\end{aligned}
$$

Since the connection $\nabla$ is torsion-free, we get $\nabla_{X} Y-\nabla_{Y} X-[X, Y]=0$, which implies that

$$
\begin{aligned}
& \alpha_{21}(X, Y)-\alpha_{21}(Y, X)-\beta_{1}([X, Y])=0, \\
& \alpha_{22}(X, Y)-\alpha_{22}(Y, X)-\beta_{2}([X, Y])=0, \\
& \alpha_{23}(X, Y)-\alpha_{23}(Y, X)-\beta_{3}([X, Y])=0 .
\end{aligned}
$$

Moreover, by symmetry, we have

$$
\alpha_{11}(X, Y)-\alpha_{11}(Y, X)=0
$$

and

$$
\alpha_{14}(X, Y)-\alpha_{14}(Y, X)=0 .
$$

Other terms vanishes by symmetry. Namely,

$$
\begin{gathered}
\alpha_{1}(X, Y)+\alpha_{10}(X, Y)+\alpha_{18}(X, Y)-\alpha_{1}(Y, X)-\alpha_{10}(Y, X)-\alpha_{18}(Y, X)=0, \\
\alpha_{3}(X, Y)+\alpha_{6}(X, Y)-\alpha_{3}(Y, X)-\alpha_{6}(Y, X)=0,
\end{gathered}
$$

and

$$
\begin{array}{r}
\alpha_{4}(X, Y)+\alpha_{5}(X, Y)+\alpha_{15}(X, Y)+\alpha_{17}(X, Y)+\alpha_{19}(X, Y) \\
-\alpha_{4}(Y, X)-\alpha_{5}(Y, X)-\alpha_{15}(Y, X)-\alpha_{17}(X, Y)-\alpha_{19}(X, Y)=0 .
\end{array}
$$

The terms $\alpha_{2}, \alpha_{7}, \alpha_{8}$ et $\alpha_{12}$ can be combined. Indeed, if we set

$$
\alpha=\alpha_{2}+\alpha_{7}+\alpha_{8}+\alpha_{12},
$$

then

$$
\begin{aligned}
\alpha(X, Y)-\alpha(Y, X)= & \eta^{2}\left[f^{2}(Y \cdot X-X \cdot Y)+Y \cdot T \cdot X \cdot T-X \cdot T \cdot Y \cdot T\right] \cdot \varphi \\
= & \eta^{2}\left[f^{2}(Y \cdot X-X \cdot Y)+\|T\|^{2}(Y \cdot X-X \cdot Y)\right] \cdot \varphi \\
& -2 \eta^{2}(\langle X, T\rangle Y \cdot T-\langle Y, T\rangle X \cdot T) \cdot \varphi
\end{aligned}
$$

by taking $X$ and $Y$ as an orthonormal frame $\left\{e_{1}, e_{2}\right\}$, we have

$$
\begin{aligned}
\alpha\left(e_{1}, e_{2}\right)-\alpha\left(e_{2}, e_{1}\right)= & 2 \eta^{2}\left[-T_{1} e_{2}\left(T_{1} e_{1}+T_{2} e_{2}\right)+T_{2} e_{2}\left(T_{1} e_{1}+T_{2} e_{2}\right)\right] \cdot \varphi \\
& -2 \eta^{2} e_{1} \cdot e_{2} \cdot \varphi \\
= & -2 \eta^{2} \omega \cdot \varphi+2 \eta^{2}\left(T_{1}^{2} \omega \cdot+T_{1} T_{2}-T_{1} T_{2}+T_{2}^{2} \omega \cdot\right) \varphi \\
= & -2 \eta^{2} f^{2} \omega \cdot \varphi .
\end{aligned}
$$

Always for $X=e_{1}$ and $Y=e_{2}$,

$$
\alpha_{9}\left(e_{1}, e_{2}\right)+\alpha_{13}\left(e_{1}, e_{2}\right)-\alpha_{9}\left(e_{2}, e_{1}\right)-\alpha_{13}\left(e_{2}, e_{1}\right)=2 T \cdot \omega \cdot \varphi=-2 J(T) \cdot \varphi,
$$

where $J$ is the rotation of positive angle $\frac{\pi}{2}$ on $T M$. Finally, we get

$$
\begin{aligned}
\mathcal{R}\left(e_{1}, e_{2}\right) \varphi= & \frac{1}{4}\left(A e_{2} \cdot A e_{1}-A e_{1} \cdot A e_{2}\right) \cdot \varphi-2 \eta^{2} f^{2} \omega \cdot \varphi \\
& -\frac{1}{2} d^{\nabla} A\left(e_{1}, e_{2}\right) \cdot \varphi-2 J(T) \cdot \varphi .
\end{aligned}
$$


Using the Ricci identity

we have

$$
\mathcal{R}\left(e_{1}, e_{2}\right) \varphi=-\frac{1}{2} R_{1212} e_{1} \cdot e_{2} \cdot \varphi,
$$

$$
\left(R_{1212}+\operatorname{det}(A)-\kappa f^{2}\right) \omega \cdot \varphi=i\left(d^{\nabla} A\left(e_{1}, e_{2}\right)+\kappa^{2} f J(T)\right) \cdot \varphi .
$$

5.5. Proof of Theorem 3. From the preliminaries, we have seen that $i i$ ) implies $i$ ). Indeed, $\mathbb{M}^{2}$ has two orthogonal Killing spinors which give two spinors solutions of (18).

Now, we will prove that $i$ ) implies $i i$ ). So, we assume that there exists two orthogonal nontrivial spinor fields $\varphi_{1}$ and $\varphi_{2}$ solutions of (18) and that Equation (11) and (12) are satisfied. Thus, by Proposition 5.1, we have

$$
-G \cdot \overline{\varphi_{j}}=C \cdot \varphi_{j}, \text { for } j=1,2,
$$

where $G=R_{1212}+\operatorname{det}(A)-\kappa f^{2}$ and $C=d^{\nabla} A\left(e_{1}, e_{2}\right)+\kappa f J(T)$. Hence, we have

$$
C \cdot \varphi_{j}^{ \pm}= \pm G \varphi_{j}^{\mp} \text {. }
$$

We deduce easliy from 20 that $\left\langle C \cdot \varphi_{1}, \varphi_{2}\right\rangle=0$. Moreover, by assumption, $\left\langle\varphi_{1}, \varphi_{2}\right\rangle=0$, then since $\Sigma M$ is of complex rank 2, we have $C \cdot \varphi_{1}=h \varphi_{1}$, with $h$ a complex-valued function. By taking the inner product by $\varphi_{1}$, we deduce that $0=\left\langle C \cdot \varphi_{1}, \varphi_{1}\right\rangle=h\left|\varphi_{1}\right|^{2}$. Since $\varphi_{1}$ never vanishes the real part of $h$ vanishes identically. Now, from 19 , we deduce that

$$
\pm G \varphi_{1}^{\mp}=h \varphi_{1}^{\mp} .
$$

Since $\varphi_{1}^{+}$and $\varphi_{1}^{-}$do not vansish at the same point, we have that $h$ is real-valued. hence $h$ vanishes identically and thus $G$ too. Finally, from $(19)$, we deduce that $C=0$. But $G=0$ and $C=0$ are respectively the Gauss and Codazzi equations. Since we assume that Equations $(11)$ and $(12)$ are satisfied, we conclude by using Theorem 1 .

5.6. A final remark about Dirac equation. If a spinor $\varphi$ is solution of the equation of restricted Killing spinor (18), then, it also satisfies the following Dirac equation:

$$
D \varphi=i H \varphi-i \eta T \cdot \varphi-2 \eta f \varphi
$$

and the following property: for all $X \in \mathfrak{X}(M)$,

$$
X\langle\varphi, \bar{\varphi}\rangle=-2 \Re e\langle i \eta X \cdot T \cdot \varphi+i \eta<X, T>\varphi, \bar{\varphi}\rangle .
$$

Note that the right hand side of 22 vanishes if $\eta$ is real, that i for the case of $\mathbb{S}^{2} \times \mathbb{R}_{1}$.

Using computations of [14 and [9, we can prove that (18) is equivalent to 21 ) and (22). Hence, as in 14, Theorem 3 can be rewritten with two orthogonal spinors solutions of the Dirac equation (21) and satisfying condition (22).

\section{REFERENCES}

[1] C. Bär, P. Gauduchon, and A. Moroianu, Generalized cylinders in semi-Riemannian and spin geometry, Math. Z. 249 (2005), no. 3, 545-580.

[2] H. Baum, Spin-Strukturen und Dirac Operatoren über pseudo-Riemannschen Mannigfaltgkeiten, Teubner-Texte zur Mathematik, Bd. 41 Teubner-Verlag, Leipzig, 1981.

[3] H. Baum and O. Müller, Codazzi spinors and global ly hyperbolic manifolds with special holonomy, Math. Z. 258 (2008), no. 1, 185-211.

[4] M. do Carmo, Riemannian Geometry. Birkhäuser, 1992.

[5] B. Daniel, Isometric immersions into $\mathbb{S}^{n} \times \mathbb{R}$ and $\mathbb{H}^{n} \times \mathbb{R}$ and applications to minimal surfaces, Trans. Amer. Math. Soc. 361 (2009), no 12, 6255-6282.

[6] T. Friedrich, On the spinor representation of surfaces in Euclidean 3-space, J. Geom. Phys. 28 (1998), no 1-2, 143-157.

[7] R. Kusner, N. Schmitt, The spinor representation of surfaces in space, ArXiv:dg-ga/9610005.

[8] M.A. Lawn, Immersions of Lorentzian surfaces in $\mathbb{R}^{2,1}$, J. Geom. Phys. 58 (2008), no 6, 683-700. 
[9] M.A. Lawn and J. Roth, Spinorial characterizations of surfaces into 3-dimensional Lorentzian space forms, arXiv:1001.4198, submitted (2010).

[10] B. Lawson and M.-L. Michelson, Spin Geometry, Princeton University Press, 1989.

[11] B. Morel, Surfaces in $\mathbb{S}^{3}$ and $\mathbb{H}^{3}$ via spinors, Sémin. Théor. Spectr. Géom., Univ Grenbole 1, 23 (2005), 131-144.

[12] B. ONeill, Semi-riemannian geometry with applications to relativity, Academic Press, New York, 1983.

[13] H. Rosenberg, Bryant surfaces. In The global theory of minimal surfaces in flat spaces, Martina Franca, Italy 1999, Lecture Notes in Mathematics 1775. Springer, 2002.

[14] J. Roth, Spinorial characterization of surfaces into 3-dimensional homogeneous manifolds, J. Geom. Phys 60 (2010), 1045-106.

[15] K. Tenenblat On isometric immersions of Riemannian manifolds, Bol. Soc. Brasil Mat. 2 (1971) no 2, 23-36.

[16] T.J. Willmore, Riemannian Geometry, The Clarendon Press, Oxford University Press, New York, 1993.

(J. Roth) LAMA, Université Paris-Est - Marne-la-Vallée, 5 Bd Descartes, Cité Descartes, ChampsSUR-Marne, F-77454 MaRnE-LA-VALLÉE

E-mail address: Julien.Roth@univ-mlv.fr 